\title{
Aislamiento y caracterización molecular y bioquímica de una cianobacteria (Anabaena sp.) del río Amazonas, Loreto-Perú
}

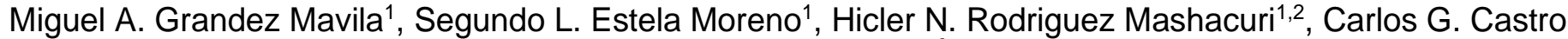 \\ Cobos $^{1,2}$, Milagros M. Torres Vásquez ${ }^{1}$, Jorge L. Marapara del Águila², Juan C. Castro Gómez², Marianela \\ Cobos Ruiz ${ }^{1}$ \\ ${ }^{1}$ Laboratorio de Biotecnología y Bioenergética (LBB) - Universidad Científica del Perú (UCP), Av. José \\ Abelardo Quiñones Km 2.5, San Juan Bautista, lquitos, Perú \\ 2Unidad Especializada del Laboratorio de Investigación de Biotecnología (UELIB), Centro de Investigación de \\ Recursos Naturales de la UNAP (CIRNA)-Universidad Nacional de la Amazonia Peruana (UNAP), Psje. Los \\ Paujiles S/N, San Juan Bautista, lquitos, Perú
}

Recibido el 15 de junio del 2021. Aceptado el 29 de junio 2021

DOI: https://doi.org/10.33017/RevECIPeru2021.0005/

\section{Resumen}

Las cianobacterias son procariotas fotosintéticos con distribución cosmopolita. Debido a los múltiples compuestos bioactivos que producen y las diversas aplicaciones potenciales que pueden tener, han llamado la atención de la comunidad científica. Sin embargo, aún existen vacíos en el conocimiento científico básico de las cianobacterias y por ende tienen limitado uso biotecnológico. Por tanto, para ampliar los conocimientos científicos de estos microorganismos, la presente investigación tuvo como principal objetivo aislar y caracterizar a nivel molecular y bioquímico la cianobacteria Anabaena sp. del río Amazonas. Las muestras de agua fueron colectadas del río Amazonas y se enriquecieron con medio ASM1. Luego, se aisló y cultivó con métodos estándares una especie de cianobacteria. Posteriormente, la cianobacteria aislada fue identificada morfológicamente y caracterizada a nivel molecular y bioquímico. Los análisis morfológicos a nivel microscópico y su caracterización molecular indican que se aisló la cianobacteria Anabaena sp. El análisis bioquímico muestra que la cianobacteria tiene $28,40 \%$ de proteínas, $10,08 \%$ de lípidos, 9,34\% de carbohidratos y $11,20 \%$ de cenizas. En conclusión, se ha aislado y cultivado de manera exitosa la cianobacteria Anabaena sp. la misma que puede tener potenciales aplicaciones para la producción biotecnológica de compuestos nutritivos para humanos.

Descriptores: Biodiversidad, biología molecular, cianobacteria, ecosistema amazónico

\section{Abstract}

Cyanobacteria are photosynthetic prokaryotes with a cosmopolitan distribution. Due to the multiple bioactive compounds that produce and their diverse potential applications, these microorganisms have attracted the attention of the scientific community. However, there are still gaps in the basic scientific knowledge of cyanobacteria and thus have limited biotechnological use. Therefore, in order to expand the scientific knowledge of these microorganisms, the main objective of the present research was to isolate and characterize at the molecular and biochemical level the cyanobacteria Anabaena sp. from the Amazon River. Water samples were collected from the Amazon river and enriched with ASM1 medium. Then, a cyanobacteria species was isolated and cultured with standard methods. Subsequently, the isolated cyanobacteria was morphologically identified and characterized at the molecular and biochemical levels. The morphological analysis at the microscopic level and its molecular characterization indicate that the cyanobacteria Anabaena sp. was isolated. The biochemical analysis shown that the cyanobacteria has $28.40 \%$ of proteins, $10.08 \%$ of lipids, $9.34 \%$ of carbohydrates, and 
$11.20 \%$ of ashes. In conclusion, the cyanobacteria Anabaena sp. has been successfully isolated and cultured and may have potential applications for the biotechnological production of human nutritional compounds.

Keywords: Biodiversity, molecular biology, cyanobacteria, Amazonian ecosystem

\section{Introducción}

Actualmente, los cuerpos de agua dulce constituyen un recurso natural invaluable en diversos aspectos tales como económico, cultural, estético, educativo y científico. Sin embargo, estos ecosistemas están experimentando cambios en su biodiversidad, calidad y cantidad por diversas actividades humanas que ocasionan contaminación, destrucción y degradación de hábitats [1].

La región nororiental del Perú, particularmente la región de Loreto, contiene una gran variedad de ambientes acuáticos de agua dulce (es decir, ríos, lagunas, etc.) que albergan una rica biodiversidad. Las investigaciones enfocadas en la identificación y caracterización de las microalgas y cianobacterias deben ser un esfuerzo continuo, debido a que solo unos pocos miles $(\sim 50,000)$ se conservan en colecciones de cultivos de microalgas en todo el mundo y de ellos, solo unos pocos cientos $(<10 \%)$ han sido investigados por su composición bioquímica [2].

En la Amazonia peruana existen algunos estudios de identificación taxonómica y molecular de microalgas [2] y sólo un reporte sobre el aislamiento y caracterización bioquímica de dos cianobacterias [3]. Consecuentemente, es necesario continuar realizando investigaciones básicas y conocer los posibles usos biotecnológicos de estos microorganismos [4]. Por tanto, el objetivo principal de esta investigación fue aislar y caracterizar a nivel molecular y bioquímico la cianobacteria Anabaena sp. del río Amazonas.

\section{Metodología}

\subsection{Colecta de muestras biológicas y enriquecimiento inicial}

La muestra de agua superficial (hasta $\sim 20 \mathrm{~cm}$ profundidad) se obtuvo del río Amazonas (0330'31,2" S, 7303'29,4" O). Estas fueron concentradas $(\sim 500 \mathrm{~mL})$ mediante filtración por arrastre con una red fitoplanctónica de $20 \mu \mathrm{m}$ de diámetro de poro [5] y transportadas protegidas de la luz y en refrigeración al LBB-UCP.
Luego, para enriquecer los cultivos, $500 \mu \mathrm{L}$ de la muestra se transfirió a microtubos de $2 \mathrm{~mL}$ y se agregó $500 \mu \mathrm{L}$ del medio de cultivo ASM1 y cultivó bajo las siguientes condiciones: fotoperiodo de 12h:12h (luz:oscuridad), temperatura de $25 \pm 1{ }^{\circ} \mathrm{C}$, intensidad de luz de $80 \mu \mathrm{Em}^{-2} . \mathrm{s}^{-1}$ con luz blanca fluorescente y agitación continua a 100 rpm por 4 semanas.

\subsection{Aislamiento, cultivo y cosecha de las cianobacterias}

Después del cultivo inicial, las cianobacterias fueron aisladas utilizando las técnicas de lavado celular y de dilución en placas con agar [6]. A medida que la biomasa de los cultivos cianobacterianos aumentaron se transfirieron a matraces de Erlenmeyer y los cultivos cianobacterianos se mantuvieron bajo las condiciones de cultivo indicadas por varias semanas [2].

Posteriormente, las células se cosecharon por centrifugación (4500 rpm a $4^{\circ} \mathrm{C}$ por $\left.10 \mathrm{~min}\right)$. Una fracción de la biomasa fresca $(100 \mathrm{mg})$ se empleó para purificar el ADN genómico para su caracterización molecular y el resto de la biomasa se secó en una estufa a $70^{\circ} \mathrm{C}$ por $72 \mathrm{~h}$ para realizar la caracterización bioquímica.

\subsection{Caracterización molecular}

El ADN genómico fue purificado de acuerdo a Cobos et al. [2] con algunas modificaciones. Luego, la calidad y cantidad del ADN genómico purificado se realizó mediante análisis espectrofotométrico empleando un espectrofotómetro UV/Vis NanoDrop 2000 y análisis electroforético en gel de agarosa al $1 \%$ [5]. Luego el ADN genómico fue amplificado mediante la reacción en cadena de la polimerasa (RCP) según Valerio et al. [7], usando cebadores específicos para cianobacterias que hibridan en el gen ribosomal 16S (CYA738F: 5'ATACCCCWGTAGTCCTAGC-3', CYAN1281R: 5'GCAATTACTAGCGATTCCTCC-3'). Las condiciones de la RCP se llevaron a cabo de acuerdo a Boutte et al. [8] con algunas modificaciones en las temperaturas de hibridación $\left(55^{\circ} \mathrm{C}\right)$ y elongación $\left(72^{\circ} \mathrm{C}\right)$ por 35 ciclos. 
Los amplicones fueron resueltos en gel de agarosa al $2 \%$ y visualizados con un transiluminador de luz UV y registrados fotográficamente.

\subsection{Prueba de la axenicidad de los cultivos}

Para demostrar que los cultivos de la cianobacteria eran axénicos (ausencia de contaminación con microalgas) se procedió a amplificar el ADN genómico purificado siguiendo el protocolo de Cobos et al. [2] usando cebadores que hibridan en sitios flanqueantes de la región ITS2 (ITS2F: 5'GAGCATGTCTGCCTCAGC-3', ITS2R: 5'GGTAGCCTTGCCTGAGC-3') y permiten amplificar una región de $\sim 300$ pb siempre que exista ADN genómico de microalgas, (p.ej., clorofitas), protozoos y/o de hongos. Se empleó como controles positivos de amplificación el ADN genómico purificado de las microalgas Ankistrodesmus falcatus y Chlalmydomonas reinhardtii. Los amplicones obtenidos fueron resueltos en gel de agarosa al $2 \%$ y visualizados en un transiluminador de luz UV y registrados fotográficamente.

\subsection{Caracterización bioquímica}

Se determinó el contenido de lípidos, carbohidratos, proteínas y cenizas. La extracción de lípidos totales se realizó según Yu et al. [9], la determinación de proteínas se realizó por triplicado de acuerdo al protocolo descrito por Hartree et al.[10], para la cuantificación de carbohidratos se aplicó el método de Dubois et al. [11] previa hidrólisis ácida con $\mathrm{HCl}$ $2 \mathrm{~N}$ y el contenido de cenizas se determinó de acuerdo a Leenin et al. [12]

\section{Resultados y discusión de los resultados}

\subsection{Aislamiento y cultivo de Anabaena sp.}

En el presente trabajo de investigación se logró aislar y cultivar en condiciones de laboratorio la cianobacteria Anabaena sp. de la Amazonia peruana (Figura 1). El éxito en este proceso lo podemos atribuir a los métodos de aislamiento de cianobacterias implementados en base a los protocolos descritos previamente por Rippka, Allen y Castenholz [13]-[15]. Con estas aproximaciones metodológicas ha sido posible aislar la cianobacteria filamentosa, tal como ha sido reportado previamente [16]-[18]. Por lo tanto, el presente trabajo de investigación corrobora que los métodos de aislamiento publicados son efectivos para el aislamiento de cianobacterias nativas de la Amazonía peruana.
Interesantemente, con las aproximaciones metodológicas empleadas se logró obtener cultivos unialgales de Anabaena sp. y fueron axénicos, es decir libre de contaminación con microorganismos eucariotas (p.ej., microalgas, protozoos, hongos, etc.). Para lograr esta condición, no fue necesario el uso de antibióticos o inhibidores del crecimiento de microorganismos eucariotas (p.ej., cicloheximida, imipenem, etc.) empleados por otros investigadores [19], [20]. Consecuentemente, la cianobacteria aisladas es apropiada para futuros estudios ómicos (p.ej., genómico, transcriptómico, proteómico, metabolómico, lipidómica, alimentómica, etc.) tal como ha sido reportado para otras cianobacterias alrededor del mundo [21]-[24].

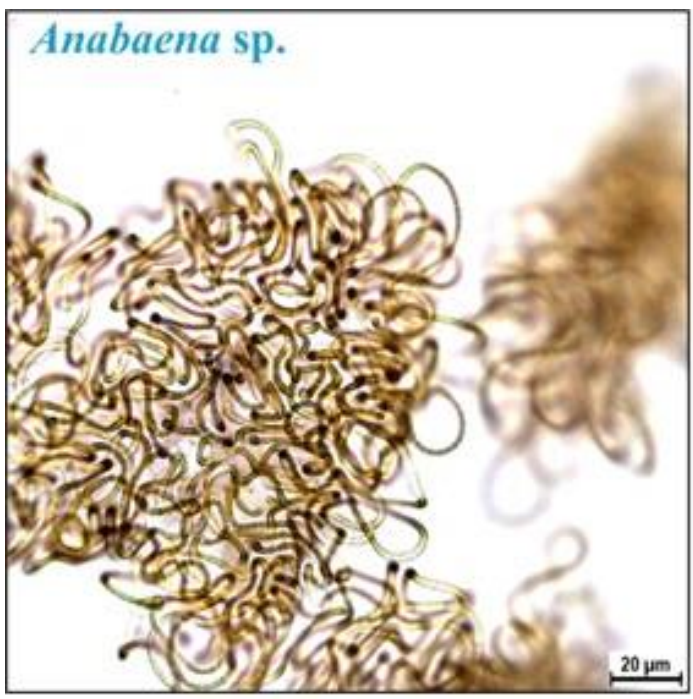

Figura 1. Microfotografía que muestran las características morfológicas de Anabaena sp.

\subsection{Caracterización molecular}

Con el protocolo estandarizado se ha logrado purificar el ADN genómico de Anabaena sp. EI ADN purificado se caracterizó por su elevada calidad con ratios $A_{260} / A_{230}$ de 2,1 y de $A_{260} / A_{280} 1,9$.

En base a las características morfológicas evidenciadas en las observaciones microscópicas se pudo reconocer que la cianobacteria aislada correspondía al género Anabaena (Figura 2). Sin embargo, para corroborar estas observaciones se emplearon herramientas moleculares, primero para purificar el ADN genómico y luego amplificar una región específica del gen $16 \mathrm{~S}$ presente en las cianobacterias. El ADN purificado se caracterizó por su alta calidad y cantidad apropiadas para los análisis moleculares (Figura 3). De acuerdo a Green et al. [25] los ratios de calidad obtenidos sugieren ausencia de contaminantes que son comunes en las 
muestras de ADN genómico, tales como proteínas, polisacáridos y otros metabolitos. Adicionalmente, la amplificación exitosa del gen ribosomal $16 \mathrm{~S}$ nos indica claramente que se aisló una cianobacteria, toda vez que el operón del ARNr y en particular el gen ribosomal 16S está presente en las cianobacterias y otros procariotas tal como lo indican otros autores [26], [27].

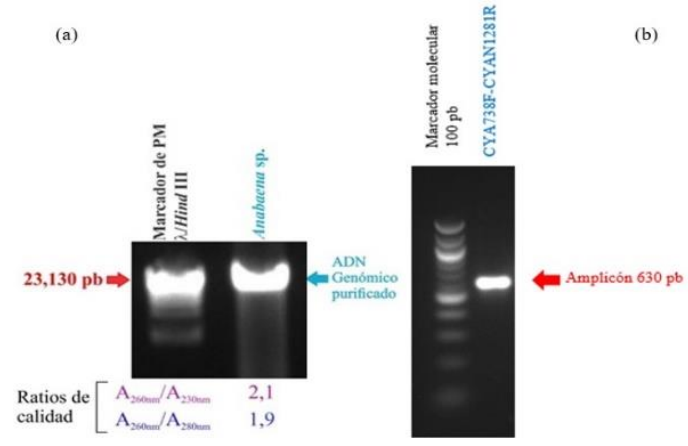

Figura 2. Caracterización molecular (a) análisis electroforético y espectrofotométrico del ADN genómico purificado de Anabaena sp. (b) análisis electroforético de los productos de RCP con cebadores específicos de cianobacterias.

Consecuentemente, la amplificación y por ende la identificación de esta región es empleada frecuentemente para identificar estos microorganismos [28], [29]. Sin embargo, para una identificación taxonómica a nivel de especie será necesario, en una siguiente etapa de nuestra investigación, secuenciar estas regiones amplificadas.

Asimismo, en base a las características morfológicas y la amplificación exitosa mediante la técnica de RCP del gen ARNr 16S de la muestra de ADN genómico de Anabaena sp. aisladas corresponden al grupo de las cianobacterias

\subsection{Axenicidad del cultivo de Anabaena sp.}

La axenicidad del cultivo de la cianobacteria se corroboró mediante la técnica de la RCP empleando cebadores específicos para la región ITS2, propia de microrganismos eucariotas que podrían contaminar los cultivos (p.ej., microalgas, hongos, u otros microorganismos eucariotas). Los resultados muestran que el cultivo es axénico, toda vez que no mostraron amplicones correspondientes a la región ITS2, que sí se observa en los controles positivos, constituidos por ADN genómico de dos especies de microalgas (Ankistrodesmus falcatus y Chlamydomonas reinhardtii).

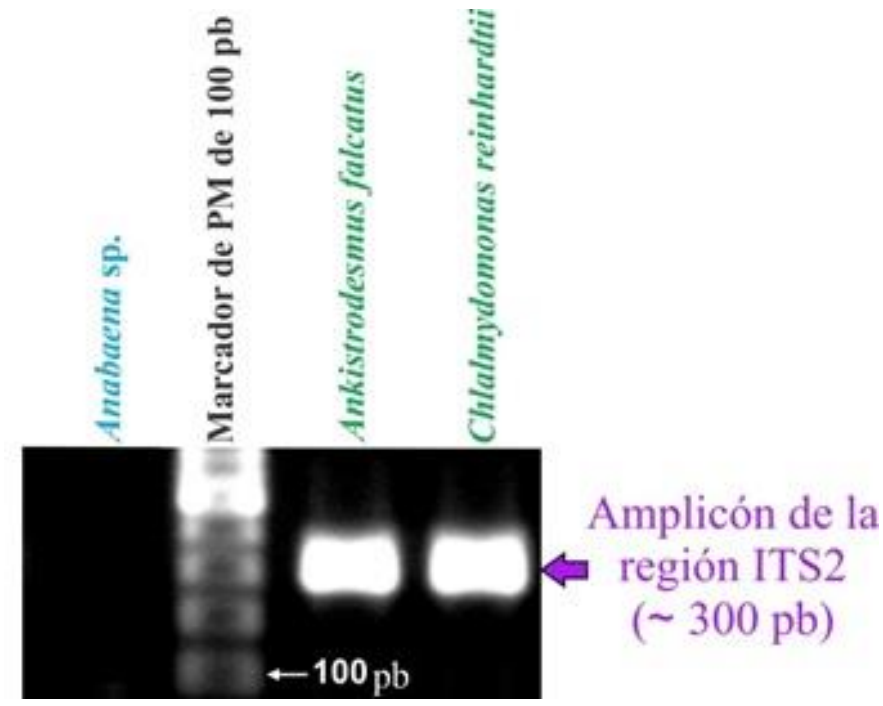

Figura 3. Análisis electroforético de los amplicones obtenidos después de amplificar las muestras de ADN genómico mediante la RCP empleando los cebadores ITS2F y ITS2R.

\subsection{Caracterización bioquímica}

Estos resultados están respaldados por otras investigaciones a nivel bioquímico de otras especies de cianobacterias como Anabaena spp., Arthrospira spp., Oscillatoria spp., Synechocystis spp, etc. [30][32]. Cabe resaltar, Anabaena sp. sobresalió por el alto contenido de proteínas, en comparación con otros componentes bioquímicos analizados. Probablemente, una importante fracción de estas proteínas cianobacterianas están constituidas por las ficocianinas, proteínas comúnmente presentes en estos microorganismos [33]-[35] y que cumplen roles fundamentales en la fotosíntesis y la eliminación de radicales libres por su potente actividad antioxidante [35]-[38]. En las siguientes etapas de análisis bioquímicos y moleculares de este género de cianobacteria aislada se deberá identificar estas proteínas y otros compuestos bioactivos con potencial nutracéutico y biotecnológico aplicables en los distintos campos tales como, farmacéutica, nutracéutica, acuicultura, agronomía, biorremediación, etc.

\section{Conclusiones.}

Se ha logrado aislar con éxito y cultivar en condiciones de laboratorio a la cianobacteria Anabaena sp. proveniente del rio Amazonas. Esta cianobacteria fue identificada morfológica $y$ molecularmente. Asimismo, su caracterización bioquímica indica que puede ser una importante fuente de nutrientes para humanos, particularmente por su alto contenido de proteínas, que podría aprovecharse como suplemento alimenticio. Es necesario continuar realizando investigaciones 


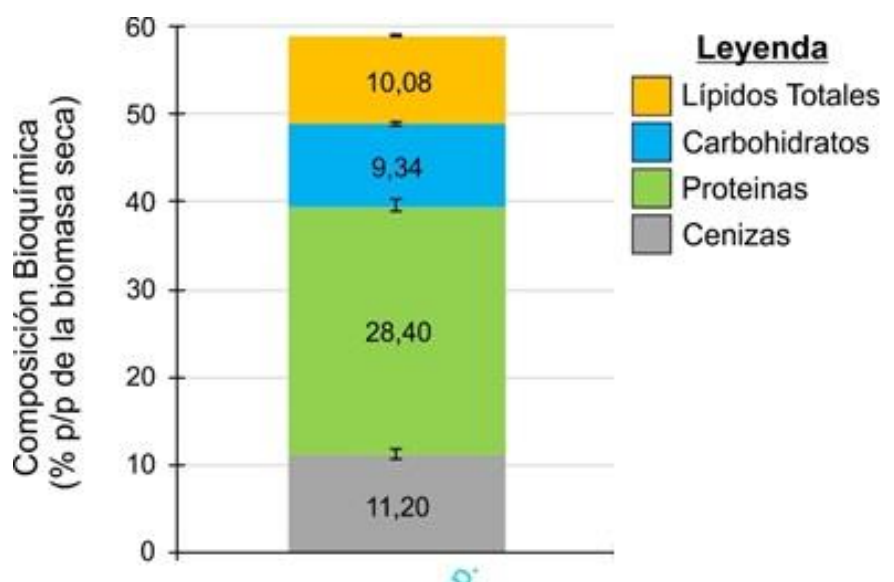

Figura 4. Contenido de Lípidos totales, carbohidratos, proteínas y cenizas de Anabaena sp.

ómicas, como el metaboloma de esta especie, debido a que las cianobacterias en general están catalogadas como biofábricas de metabolitos primarios y secundarios y así nos puede revelar las múltiples aplicaciones biotecnológicas que pueden tener estos microorganismos.

\section{Agradecimientos}

Al proyecto Adquisición de un Sistema de Secuenciamiento masivo Next Seq 550 como soporte para estudios genómicos y metagenómicos de la biodiversidad de la Amazonía Peruana. Convenio 018-2018-FONDECYT-BM-INV-INC

\section{Referencias}

[1] H. Ortega, L. Chocano, C. Palma, y I. Samanez, «Biota acuática en la Amazonía Peruana: diversidad y usos como indicadores ambientales en el Bajo Urubamba (Cusco Ucayali)», Rev. Peru. Biol., vol. 17, n. ${ }^{\circ}$, may 2011, doi: 10.15381/rpb.v17i1.47.

[2] M. Cobos et al., «Isolation and Characterization of Native Microalgae from the Peruvian Amazon with Potential for Biodiesel Production», Energies, vol. 10, p. 224, feb. 2017, doi: 10.3390/en10020224.

[3] L. V. Diaz Calampa y M. A. Urcia Saboya, «Aislamiento e Identificación de Microalgas Oleaginosas en las Cuencas de los Rios Amazonas, Itaya y Nanay-2013», Universidad Cientifica del Perú, lquitos-Perú, 2014.
[4] J. Roset, S. Aguayo, y M. Muñoz, «Detección de cianobacterias y sus toxinas. Una revisión», Rev. Toxicol, vol. 18, pp. 65-71, 2001.

[5] M. Cobos, J. Castro, y G. Vargas, Protocolos para el aislamiento, caracterización bioquímica y molecular de microalgas oleaginosas. lquitos-Perú, 2016.

[6] A. Richmond, Handbook of microalgal culture: biotechnology and applied phycology. Blackwell Science, 2004. Accedido: oct. 05, 2018. [En línea]. Disponible en: http://gen.lib.rus.ec/book/index.php?md5=9e0 74d250deaae72b8c04aeb8cb9a559

[7] E. Valério, L. Chambel, S. Paulino, N. Faria, P. Pereira, y R. 2009 Tenreiro, «Molecular identification, typing and traceability of cyanobacteria from freshwater reservoirs", Microbiology, vol. 155, n. ${ }^{\circ}$ 2, pp. 642-656, doi: 10.1099/mic.0.022848-0.

[8] C. Boutte, S. Grubisic, P. Balthasart, y A. Wilmotte, «Testing of primers for the study of cyanobacterial molecular diversity by DGGE», J. Microbiol. Methods, vol. 65, n. ${ }^{\circ} 3$, pp. 542550, jun. 2006, doi: 10.1016/j.mimet.2005.09.017.

[9] X. Yu et al., "Isolation of a novel strain of Monoraphidium sp. and characterization of its potential application as biodiesel feedstock», Bioresour. Technol., vol. 121, pp. 256-262, oct. 2012, doi: 10.1016/j.biortech.2012.07.002.

[10] E. F. Hartree, «Determination of protein: A modification of the lowry method that gives a linear photometric response», Anal. Biochem., vol. 48, n. ${ }^{\circ} 2$, pp. 422-427, ago. 1972, doi: 10.1016/0003-2697(72)90094-2.

[11] Michel. DuBois, K. A. Gilles, J. K. Hamilton, P. A. Rebers, y Fred. Smith, "Colorimetric Method for Determination of Sugars and Related Substances», Anal. Chem., vol. 28, n. ${ }^{\circ}$ 3 , pp. 350-356, mar. 1956, doi: 10.1021/ac60111a017.

[12] L. Flores, A. Ruiz, y A. Oscanoa, «Protocolo para determinación de cenizas en microalgas liofilizadas», vol. 48, n. ${ }^{\circ} 1$, p. 3, 2021.

[13] Rippka R., "Isolation and purification of cyanobacteria.», Methods Enzymol, vol. 167, pp. 3-27., 1988.

[14] M. M. Allen, «Simple Conditions for Growth of Unicellular Blue-Green Algae on Plates 1, 2», J. Phycol., vol. 4, n. ${ }^{\circ}$ 1, pp. 1-4, 1968, doi: 10.1111/j.1529-8817.1968.tb04667.x.

[15] R. W. Castenholz, "[3] Culturing methods for cyanobacteria", en Methods in Enzymology, vol. 167, Academic Press, 1988, pp. 68-93. doi: 10.1016/0076-6879(88)67006-6. 
[16] V. S. Uma, D. Gnanasekaran, U. Lakshmanan, y $P$. Dharmar, «Survey and isolation of marine cyanobacteria from eastern coast of India as a biodiesel feedstock», Biocatal. Agric. Biotechnol., vol. 24, p. 101541, mar. 2020, doi: 10.1016/j.bcab.2020.101541.

[17] T. S. Thilak, P. V. Madhusoodanan, N. S. Pradeep, y R. Prakashkumar, «Isolation and taxonomy of the blue-green algae (Cyanobacteria), Nostoc and Anabaena in Kerala State, India», Acta Bot. Hung., vol. 62, n. ${ }^{\circ} 1-2$, pp. 163-174, mar. 2020, doi: 10.1556/034.62.2020.1-2.10.

[18] N. B. Prihantini, «Morphological identification, isolation, and culturing of cyanobacteria derived from hot spring of Cisolok and Galunggung Mountain based on enrichment method», J. Phys. Conf. Ser., vol. 1442, p. 012069, ene. 2020, doi: 10.1088/17426596/1442/1/012069.

[19] L. Sena, D. Rojas, E. Montiel, H. González, J. Moret, y L. Naranjo, «A strategy to obtain axenic cultures of Arthrospira spp. cyanobacteria", World J. Microbiol. Biotechnol., vol. 27, n. ${ }^{\circ}$ 5, pp. 1045-1053, may 2011, doi: 10.1007/s11274-010-0549-6.

[20] T. Vaara, M. Vaara, y S. Niemelä, «Two Improved Methods for Obtaining Axenic Cultures of Cyanobacteria», Appl. Environ. Microbiol., vol. 38, n. ${ }^{\circ}$ 5, pp. 1011-1014, nov. 1979.

[21] D. Schwarz, I. Orf, J. Kopka, y M. Hagemann, «Recent Applications of Metabolomics Toward Cyanobacteria», Metabolites, vol. 3, n. ${ }^{\circ}$ 1, Art. n. ${ }^{\circ}$ 1, mar. 2013, doi: 10.3390/metabo3010072.

[22] T. Nakajima et al., "Integrated metabolic flux and omics analysis of Synechocystis sp. PCC 6803 under mixotrophic and photoheterotrophic conditions», Plant Cell Physiol., vol. 55, n. ${ }^{\circ}$ 9, pp. 1605-1612, sep. 2014, doi: 10.1093/pcp/pcu091.

[23] W.-R. Lin, S.-I. Tan, C.-C. Hsiang, P.-K. Sung, y I.-S. Ng, "Challenges and opportunity of recent genome editing and multi-omics in cyanobacteria and microalgae for biorefinery", Bioresour. Technol., vol. 291, p. 121932, nov. 2019, doi: 10.1016/j.biortech.2019.121932.

[24] Y. Yang, J. Feng, T. Li, F. Ge, y J. Zhao, "CyanOmics: an integrated database of omics for the model cyanobacterium Synechococcus sp. PCC 7002», Database, vol. 2015, n. ${ }^{\circ}$ bau127, ene. 2015, doi: 10.1093/database/bau127.

[25] M. R. Green, J. Sambrook, y J. Sambrook, Molecular cloning: a laboratory manual, 4th ed.
Cold Spring Harbor, N.Y: Cold Spring Harbor Laboratory Press, 2012.

[26] B. A. Neilan, B. P. Burns, D. A. Relman, y D. R. Lowe, «Molecular Identification of Cyanobacteria Associated with Stromatolites from Distinct Geographical Locations», Astrobiology, vol. 2, n. ${ }^{\circ}$ 3, pp. 271-280, ago. 2002, doi: 10.1089/153110702762027853.

[27] F. Pomati et al., «The Freshwater Cyanobacterium Planktothrix Sp. Fp1: Molecular Identification and Detection of Paralytic Shellfish Poisoning Toxins», J. Phycol., vol. 36, n. ${ }^{\circ} 3$, pp. 553-562, 2000, doi: 10.1046/j.1529-8817.2000.99181.x.

[28] C. Lyra et al., «Molecular characterization of planktic cyanobacteria of Anabaena, Aphanizomenon, Microcystis and Planktothrix genera», International Journal of Systematic and Evolutionary Microbiology, vol. 51, pp. 513-526, 2001.

[29] J. Komárek, «Cyanobacterial Taxonomy: Current Problems and Prospects for the Integration of Traditional and Molecular Approaches», Algae, vol. 4, n. ${ }^{\circ}$ 21, pp. 349375, 2006.

[30] T. Kawaguchi y A. W. Decho, «Biochemical Characterization of Cyanobacterial Extracellular Polymers (EPS) from Modern Marine Stromatolites (Bahamas)», Prep. Biochem. Biotechnol., vol. 30, n. ${ }^{\circ} 4$, pp. 321330, ene. 2000, doi: $10.1080 / 10826060008544971$.

[31] K. Okajima et al., «Biochemical and Functional Characterization of BLUF-Type Flavin-Binding Proteins of Two Species of Cyanobacteria», J. Biochem. (Tokyo), vol. 137, n. ${ }^{\circ}$ 6, pp. 741-750, jun. 2005, doi: 10.1093/jb/mvi089.

[32] M. Cobos et al., "Nutritional evaluation and human health-promoting potential of compounds biosynthesized by native microalgae from the Peruvian Amazon», World J. Microbiol. Biotechnol., vol. 36, n. ${ }^{\circ} 8$, p. 121, jul. 2020, doi: 10.1007/s11274-020-02896-1.

[33] A. Patel, S. Mishra, R. Pawar, y P. K. Ghosh, «Purification and characterization of CPhycocyanin from cyanobacterial species of marine and freshwater habitat», Protein Expr. Purif., vol. 40, n. ${ }^{\circ}$ 2, pp. 248-255, abr. 2005, doi: 10.1016/j.pep.2004.10.028.

[34] H. H. A. El-Baky y G. S. El-Baroty, "Characterization and bioactivity of phycocyanin isolated from Spirulina maxima grown under salt stress», Food Funct., vol. 3, n. ${ }^{\circ} 4$, pp. 381-388, mar. 2012, doi: 10.1039/C2FO10194G. 
[35] V. B. Bhat y K. M. Madyastha, «Scavenging of Peroxynitrite by Phycocyanin and

Phycocyanobilin from Spirulina platensis: Protection against Oxidative Damage to DNA», Biochem. Biophys. Res. Commun., vol. 285, n. ${ }^{\circ} 2$, pp. 262-266, jul. 2001, doi: 10.1006/bbrc.2001.5195.

[36] V. B. Bhat y K. M. Madyastha, «CPhycocyanin: A Potent Peroxyl Radical Scavenger in Vivo and in Vitro", Biochem. Biophys. Res. Commun., vol. 275, n. ${ }^{\circ}$, pp. 2025, ago. 2000, doi: 10.1006/bbrc.2000.3270.

[37] M. R. Edwards, R. MacColl, y L. E. Eisele, «Some physical properties of an unusual Cphycocyanin isolated from a photosynthetic thermophile», Biochim. Biophys. Acta BBA -

[38] M. Gantar, D. Simović, S. Djilas, W. W. Gonzalez, y J. Miksovska, «Isolation, characterization and antioxidative activity of Cphycocyanin from Limnothrix sp. strain 37-21», J. Biotechnol., vol. 159, n. ${ }^{\circ}$ 1, pp. 21-26, may 2012, doi: 10.1016/j.jbiotec.2012.02.004.

agrandez@ucp.edu.pe sestelam@ucp.edu.pe

hiclernrm177unap@unapiquitos.edu.pe gilberto castrocobos@yahoo.com mimatova 16@outlook.com jorge.marapara@unapiquitos.edu.pe juan.castro@unapiquitos.edu.pe mcobos@ucp.edu.pe Bioenerg., vol. 1276, n. ${ }^{\circ}$ 1, pp. 64-70, ago. 1996, doi: 10.1016/0005-2728(96)00032-1. 